\title{
Low-dose interleukin-2 as a novel therapeutic option for refractory paraneoplastic neurologic syndrome: a case of chronic relapsing anti-Ma2/Ta paraneoplastic myeloradiculopathy
}

\author{
Seon-Jae Ahn ${ }^{1,2}$, Han-Sang Lee ${ }^{1,2}$, Woo-Jin Lee ${ }^{1,2}$, Kon Chu $^{2}$ \\ ${ }^{1}$ Center for Hospital Medicine, Seoul National University Hospital, Seoul, Korea \\ ${ }^{2}$ Department of Neurology, Comprehensive Epilepsy Center, Seoul National University Hospital, Seoul National University College of \\ Medicine, Seoul, Korea
}

Paraneoplastic neurologic syndromes (PNS) caused by anti-Ma2/Ta antibodies have diverse presentations. Myeloradiculopathy is one anti-Ma2/ Ta-associated PNS manifestation. We report the case of a patient with chronic relapsing anti-Ma2/Ta paraneoplastic myeloradiculopathy. The patient was successfully treated with low-dose human recombinant interleukin-2, despite having chronic relapsing symptoms and a refractory response to conventional immunotherapy.

Keywords: Paraneoplastic neurological syndromes, Anti-Ma2/Ta paraneoplastic syndrome, Myeloradiculopathy, Human recombinant interleukin-2

\section{Introduction}

Paraneoplastic neurologic syndromes (PNS) include several types of neurological disorders, such as cerebellar degeneration, limbic encephalitis, encephalomyelitis, and myelopathy [1]. Most cases of PNS are immune-mediated and are associated with onconeural antibodies [2]. In close association with specific cancers, several onconeural antibodies are known to cause PNS [3]. Unlike other autoimmune diseases, some cases of PNS are refractory to classical immunotherapy [4]. Herein, we present a case of paraneoplastic myeloradiculopathy with $\mathrm{Ma} 2 / \mathrm{Ta}$ antibody that was successfully treated with low-dose interleukin-2 (IL-2). The patient recovered completely after low-dose IL-2 treatment, and other previously administered immunotherapies elicited only a partial or no therapeutic response.

\section{Case Report}

A 51-year-old woman with no relevant medical history developed paresthesia in her lower extremities 6 months before she visited our hospital. The symptoms progressed to weakness in the left leg and gait imbalance over 6 months. Neurological examination revealed spasticity of the lower extremities with left leg weakness (grade III). The weakness was more severe in the distal part (ankle dorsiflexion, plantarflexion) than in the proximal part. The deep tendon reflex (DTR) was increased in the bilateral upper and lower extremities. Sensory examination revealed a diminished sense of vibration and proprioception in both feet. No apparent sensory level was observed. Myelopathy that primarily involved the posterior column was clinically suspected. Magnetic resonance imaging (MRI) of the spinal cord showed no abnormality, and pleocytosis or protein elevation were absent on cerebrospinal fluid (CSF) analysis. Nerve con-

Received: February 26, 2021 Revised: March 18, 2021 Accepted: March 23, 2021

Correspondence: Kon Chu

Department of Neurology, Seoul National University Hospital, 101 Daehak-ro, Jongno-gu, Seoul 03080, Korea

E-mail: stemcell.snu@gmail.com

ORCID: https://orcid.org/0000-0001-5863-0302

Copyright (C) 2021 by The Korean Encephalitis and Neuroinflammation Society

This is an open access article distributed under the terms of the Creative Commons Attribution Non-Commercial License (http://creativecommons.org/licenses/by-nc/4.0/) which permits unrestricted non-commercial use, distribution, and reproduction in any medium, provided the original work is properly cited. 
duction study and electromyography were performed to exclude motor neuron disease. Electrophysiological tests showed left lumbosacral radiculopathy (L5). Brain MRI revealed only mild small vessel disease. The oligoclonal band was negative. Transverse myelitis or demyelinating disease such as multiple sclerosis and neuromyelitis optica were included on the initial list for the differential diagnosis. However, these diseases were excluded because of clear spine and brain imaging, absent pleocytosis in CSF and the negative oligoclonal band.

The serum paraneoplastic antibody test from the extensive laboratory workup revealed the presence of anti-Ma2/Ta antibodies. No systemic tumors were found on computed tomography of the chest, abdomen, and pelvis. Intravenous immunoglobulin (IVIg) $400 \mathrm{mg} / \mathrm{kg}$ was administered for 5 days, based on the clinical diagnosis of paraneoplastic myeloradiculopathy.

Leg weakness improved clinically after two cycles of IVIg administration at a 4-week interval. Spasticity disappeared and the DTRs were normoreflexive on the follow-up neurological examination. However, despite the improvement in leg weakness, the patient continued to complain of gait imbalance. Second-line immunotherapy was implemented with four cycles of tocili- zumab (4 mg/kg) at monthly intervals. However, tocilizumab therapy did not elicit any clinical improvement.

A low-dose human recombinant IL-2 (aldesleukin) was administered after the tocilizumab therapy failure. Low-dose IL-2 (1 million IU) was injected subcutaneously for 5 days. The patient was able to walk normally without any discomfort 1 month later. Subsequently, low-dose IL-2 therapy was administered every month for more than a year.

There was a temporary interruption (6 months) in the hospital's supply of aldesleukin, which is the only recombinant IL-2 product that is commercially available in South Korea, due to a lack of inventory in early 2018. As a result, the patient was unable to receive treatment with recombinant IL-2 for 6 months. Gait disturbance, leg weakness, and sensory symptoms relapsed during this period. Follow-up spinal cord MRI revealed diffuse high-signal T2 intensities in the thoracic spinal cord (Figure 1). After resuming low-dose IL-2 treatment the symptoms improved drastically again. The spinal cord lesion became less prominent on the follow-up MRI that was performed after 4 months and the lesion completely disappeared a year later (Figure 2).

Low-dose IL-2 treatment was administered monthly due to

Figure 1 Spinal cord MRI obtained on myeloradiculopathy relapse
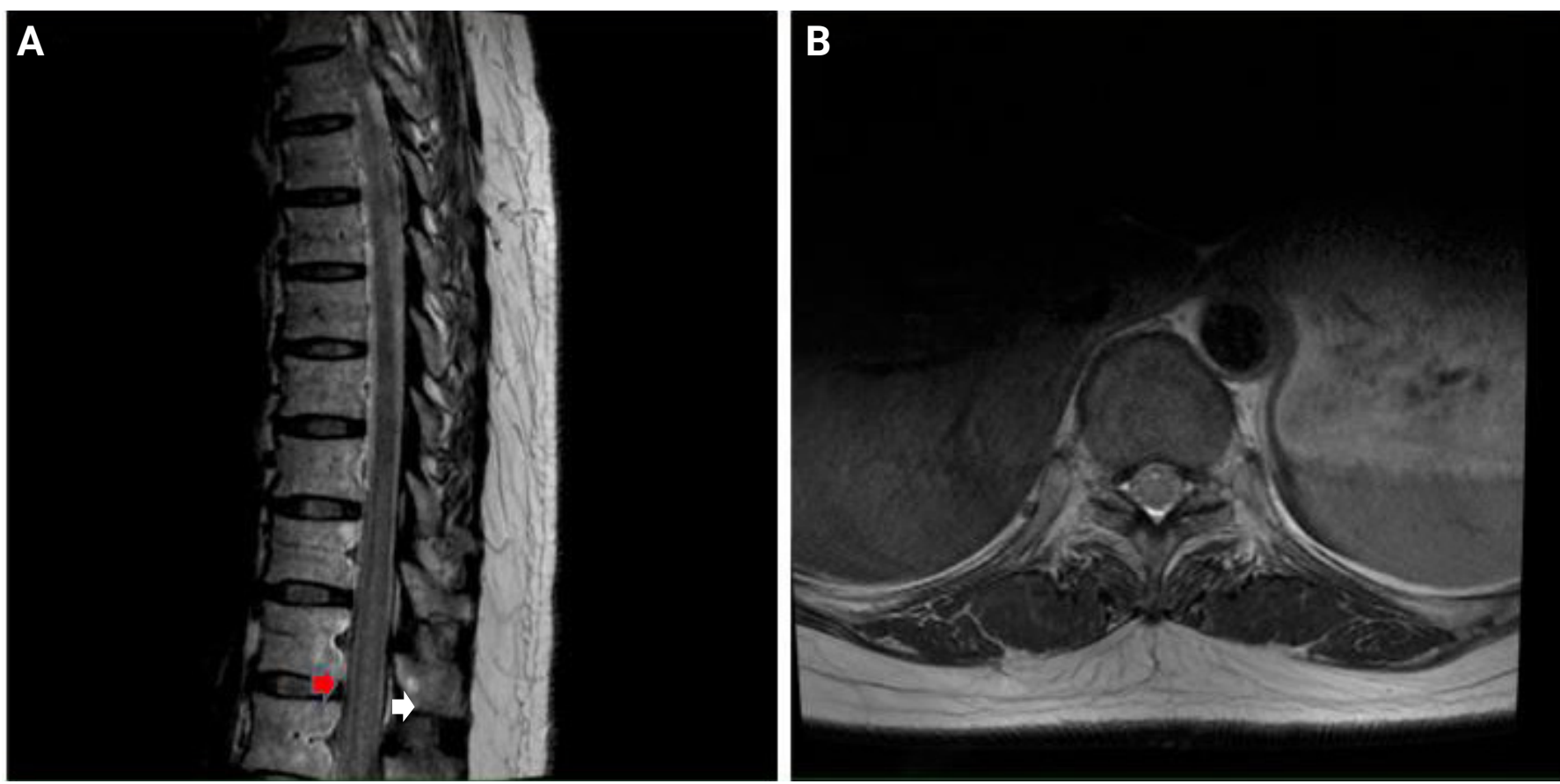

(A) Sagittal spinal MRI indicating an abnormal high-signal T2 intensity was observed in the thoracic spinal cord (arrow) during symptom relapse. (B) T9 level axial image showing diffuse high-signal T2 intensity in the spinal cord. MRI, magnetic resonance imaging. 
Figure 2 Follow-up spinal cord MRI
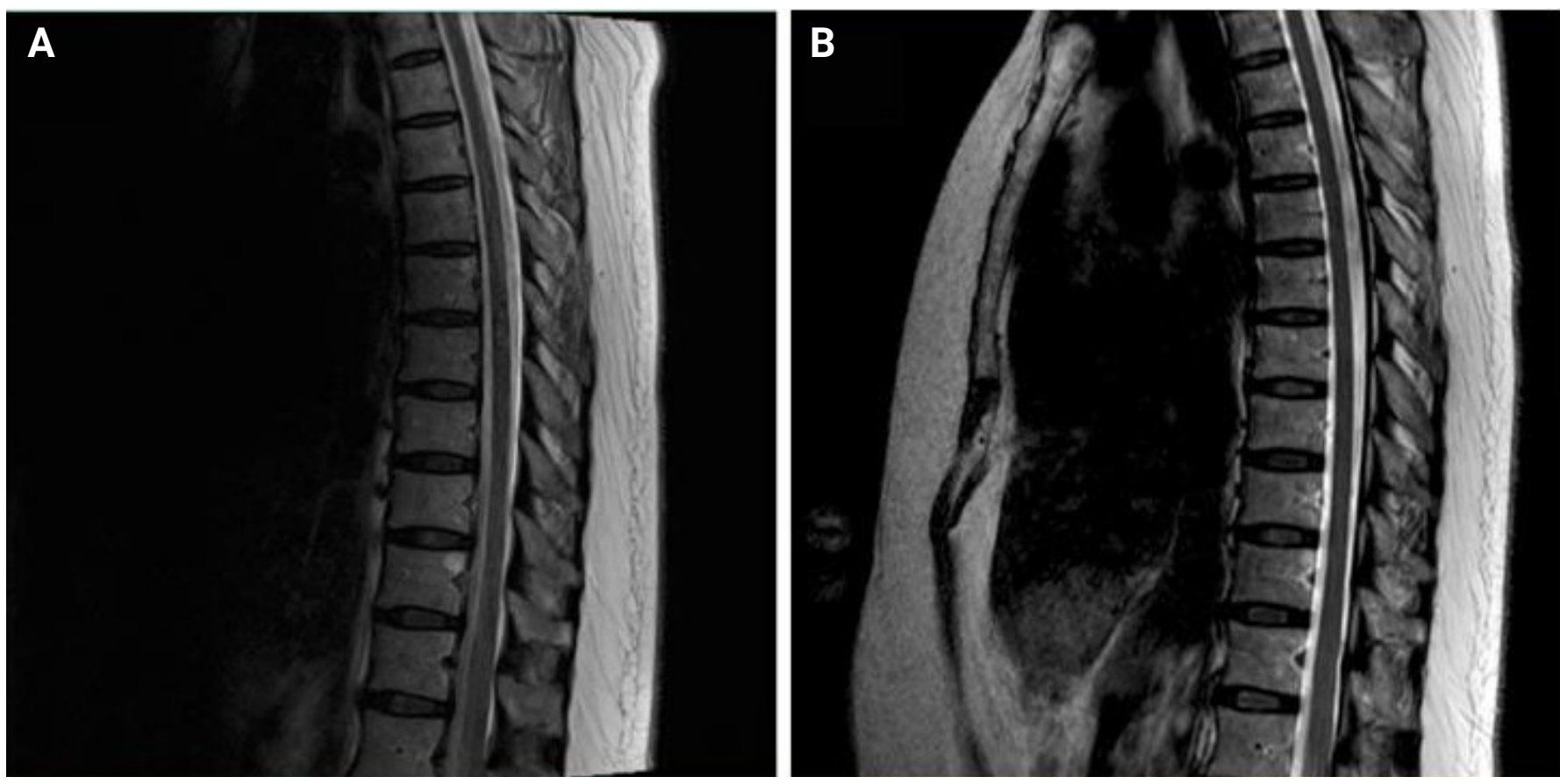

(A) Spinal cord MRI obtained 4 months after resuming low-dose interleukin-2 treatment. The abnormal high-signal T2 intensity was less prominent. (B) Complete resolution of abnormal high-signal T2 intensity on the follow-up MRI obtained after 1 year.

MRI, magnetic resonance imaging.

chronic relapsing myeloradiculopathy. Cancer screening for hidden malignancies was performed regularly along with medical treatment. The patient no longer experienced lower extremity weakness, but occasionally experienced gait disturbance if the treatment interval was prolonged.

Written informed consent was obtained for publication of this case report and accompanying images.

\section{Discussion}

Anti-Ma2/Ta paraneoplastic syndrome can manifest with diverse clinical neurologic presentations. Most cases present with limbic encephalitis or brainstem encephalitis, and cerebellar dysfunction or peripheral neuropathy have been observed in a few cases [5]. Motor neuron involvement in anti-Ma2-associated PNS has recently been reported [6]. In the article, three patients were diagnosed with myeloradiculopathy among 33 patients with anti-Ma2 antibodies. However, only two of the 38 patients presented with myelopathy in a previous clinical study of anti-Ma2-associated encephalitis [7]. To the best of our knowledge, only one other case of anti-Ma2-associated myeloradicu- lopathy has been reported and was documented in 2012 [8]. Our patient represents another rare case of paraneoplastic myeloradiculopathy associated with anti-Ma2 antibody. The immunoreaction between anti-Ma2 antibodies and Ma2 protein (paraneoplastic antigen Ma2) has been demonstrated in all neurons of the human brain, dorsal-root ganglia, intestinal autonomic neurons, and spinal cord [9]. Therefore, paraneoplastic myeloradiculopathy associated with anti-Ma2 antibody has a plausible pathophysiological mechanism.

The underlying immune mechanism for PNS is related to autoantibody production and these antibodies can be classified into two broad categories [3]. The first group consists of onconeural antibodies, which target intracellular neuronal antigens expressed by tumors. The second group consists of antibodies that target the neuronal cell surface or synapse. When compared with synaptic antibodies, onconeural antibodies induce irreversible cell damage that is mediated by cytotoxic T-cells. This may explain the poor response of onconeural PNS to immunotherapy [10]. The imbalance in the circulating T-lymphocyte subsets was demonstrated in anti-Hu antibody PNS by a previous study [11]. 
Figure 3 Clinical course and treatment response

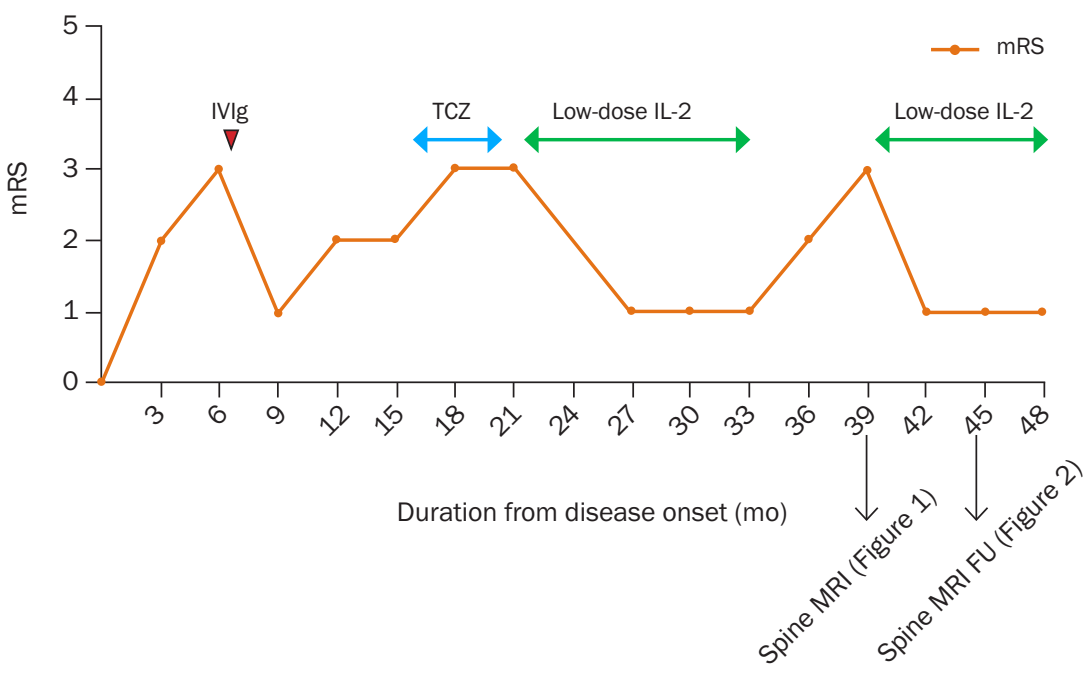

Graph of clinical course expressed in the modified Rankin Scale (mRS). Intravenous immunoglobulin (IVlg) was clinically effective but myeloradiculopathy relapsed. Tocilizumab (TCZ) showed no clinical benefit. Monthly interval low-dose interleukin-2 (IL-2) showed clinical effectiveness. However, myeloradiculopathy relapsed both clinically and radiologically during the discontinuation of the treatment.

MRI, magnetic resonance imaging; FU, follow-up.

Tani et al. [12] demonstrated that regulatory $\mathrm{T}$ cell $\left(\mathrm{T}_{\text {reg }}\right)$ dysfunction plays a key role in PNS development. They included anti-Hu and anti-Yo-positive patients with PNS and analyzed messenger RNA (mRNA) expression in the peripheral blood. The results showed downregulation of forkhead box P3 (FOXP3), tumor growth factor- $\beta$ (TGF- $\beta$ ), and cytotoxic T-lymphocyte-associated protein 4 (CTLA-4) mRNA, which are functional $\mathrm{T}_{\text {reg }}$ molecules in patients with PNS. This study suggests that a therapeutic mechanism of potential drugs could be based on $\mathrm{T}_{\text {reg }}$ modulation in PNS.

Low-dose IL-2 has become an essential therapeutic option for autoimmune neurological diseases $[13,14]$. The mechanism of action of low-dose IL-2 involves modulation of $\mathrm{T}_{\text {reg }}$ function [15]. High-dose IL-2 was originally used for treating tumors, such as melanoma or renal cell carcinoma [16]. However, high-dose IL-2 treatment has occasionally been accompanied by high-risk side effects such as severe bacteremia or vascular leak syndrome [17]. In comparison, low-dose IL-2 has been used as a new therapeutic option for autoimmune diseases because the $\mathrm{T}_{\text {reg }}$ has a lower activation threshold for IL-2 compared to the effector T cells. Low-dose IL-2 administration has demonstrated favorable outcomes in several autoimmune diseases such as hepatitis $\mathrm{C}$ virus-induced vasculitis, systemic lupus erythematosus, and graft versus host disease [18].

Considering the pathomechanism of PNS and therapeutic mechanism of low-dose IL-2 together, a treatment strategy that targets $\mathrm{T}_{\text {reg }}$ seems convincing for onconeural PNS. Our case supports this hypothesis as the patient achieved complete remission of the disease following low-dose IL-2 injection. IVIg was also effective during the initial treatment but achieved only a partial clinical response. Moreover, myelopathy recurred when low-dose IL-2 treatment was stopped unwillingly. During the second severe relapse of myelopathy, an abnormally high T2 signal appeared on spinal cord MRI. Fortunately, the clinical symptoms and abnormal MRI findings improved completely with the resumption of low-dose IL-2 treatment (Figure 3). Conventional treatment options for anti-Ma2/Ta paraneoplastic syndrome include corticosteroids, cyclophosphamide, IVIg, and azathioprine [19]. Additionally, we suggest that low-dose IL-2 could be a novel treatment option for refractory PNS.

The accuracy of the diagnosis could be debated since no accompanying tumor was found. However, according to the recommended diagnostic criteria of PNS, a diagnosis of PNS does not require the presence of a tumor [20]. According to the proposed diagnostic criteria of the Paraneoplastic Neurological Syndrome Euronetwork [20], this case belongs to the 'definite PNS' category. Of note, one limitation of this study was that immunotherapy has not been given to patients in conventional flow. The flow of immunotherapies and approach for this patient was determined by experienced physicians. 
Our case report has several limitations. First, the possibility of a false-positive result for the paraneoplastic antibody test cannot be excluded. In our hospital, the paraneoplastic antibody test was conducted with the immunoblot method. Concise sensitivity and specificity are unknown for this examination. According to a study by Budhram et al. [21], the positive predictive value of paraneoplastic antibody test with the immunoblotting method was only $39 \%$. Second, conventional immunotherapies such as corticosteroid or cyclophosphamide were not administered because of possible clinical side effects. Although the patient was successfully treated with low-dose IL-2, the clinical response to corticosteroids is not known. Third, because the appropriate treating period for low-dose IL-2 has not been established, it is difficult to determine a suitable plan for long-term treatment.

Our case showed that $\mathrm{T}_{\text {reg }}$ modulation using low-dose IL-2 could be a new treatment option for PNS. This approach can be used for patients that require refractory to first-line immunotherapy, although further research and clinical evidence with a larger sample size are needed.

\section{Conflicts of Interest}

Kon Chu has been a member of the editorial board of encephalitis since October 2020. He was not involved in the review process of this case report. There were no other potential conflicts of interest relevant to this article to report.

\section{Author Contributions}

Conceptualization: K Chu; Data Curation: SJ Ahn; Writing-original draft: SJ Ahn; Writing-review and editing: all authors.

\section{Acknowledgements}

This report was supported by the Ildong Pharmaceutical Co., Ltd. (No. 0620191880).

\section{References}

1. Graus F, Dalmau J. Paraneoplastic neurological syndromes. Curr Opin Neurol 2012;25:795-801.

2. Monstad SE, Knudsen A, Salvesen HB, Aarseth JH, Vedeler CA. Onconeural antibodies in sera from patients with various types of tumours. Cancer Immunol Immunother 2009;58:1795-1800.

3. Höftberger R, Rosenfeld MR, Dalmau J. Update on neurological paraneoplastic syndromes. Curr Opin Oncol 2015;27:489-495.

4. Rosenfeld MR, Dalmau J. Paraneoplastic neurologic disorders: a brief overview. Memo 2012;5:197-200

5. Ortega Suero G, Sola-Valls N, Escudero D, Saiz A, Graus F. Anti-Ma and anti-Ma2-associated paraneoplastic neurological syndromes. Neurologia 2018;33:18-27.

6. Vogrig A, Joubert B, Maureille A, et al. Motor neuron involvement in anti-Ma2-associated paraneoplastic neurological syndrome. J Neurol 2019;266:398-410.

7. Dalmau J, Graus F, Villarejo A, et al. Clinical analysis of anti-Ma2-associated encephalitis. Brain 2004;127(Pt 8):1831-1844.

8. Murphy SM, Khan U, Alifrangis C, et al. Anti Ma2-associated myeloradiculopathy: expanding the phenotype of anti-Ma2 associated paraneoplastic syndromes. J Neurol Neurosurg Psychiatry 2012;83:232233.

9. Voltz R, Gultekin SH, Rosenfeld MR, et al. A serologic marker of paraneoplastic limbic and brain-stem encephalitis in patients with testicular cancer. N Engl J Med 1999;340:1788-1795.

10. Pignolet BS, Gebauer CM, Liblau RS. Immunopathogenesis of paraneoplastic neurological syndromes associated with anti-Hu antibodies: a beneficial antitumor immune response going awry. Oncoimmunology 2013;2:e27384.

11. Zhang L, Qian W, Chen Q, Yin L, Li B, Wang H. Imbalance in circulating $\mathrm{T}$ lymphocyte subsets contributes to $\mathrm{Hu}$ antibody-associated paraneoplastic neurological syndromes. Cell Immunol 2014;290:245250.

12. Tani T, Tanaka K, Idezuka J, Nishizawa M. Regulatory T cells in paraneoplastic neurological syndromes. J Neuroimmunol 2008;196:166169.

13. Shin YW, Lee ST, Park KI, et al. Treatment strategies for autoimmune encephalitis. Ther Adv Neurol Disord 2017;11:1756285617722347.

14. Lim JA, Lee ST, Moon J, et al. New feasible treatment for refractory autoimmune encephalitis: low-dose interleukin-2. J Neuroimmunol 2016;299:107-111.

15. Klatzmann D, Abbas AK. The promise of low-dose interleukin-2 therapy for autoimmune and inflammatory diseases. Nat Rev Immunol 2015;15:283-294.

16. Rosenberg SA, Yang JC, Topalian SL, et al. Treatment of 283 consecutive patients with metastatic melanoma or renal cell cancer using high-dose bolus interleukin 2. JAMA 1994;271:907-913.

17. Krieg C, Létourneau S, Pantaleo G, Boyman O. Improved IL-2 immunotherapy by selective stimulation of IL-2 receptors on lymphocytes and endothelial cells. Proc Natl Acad Sci U S A 2010;107:11906-11911.

18. Collison J. Low-dose IL-2 therapy for autoimmune diseases. Nat Rev Rheumatol 2019;15:2.

19. Kraker J. Treatment of anti-Ma2/Ta paraneoplastic syndrome. Curr Treat Options Neurol 2009;11:46-51.

20. Graus F, Delattre JY, Antoine JC, et al. Recommended diagnostic criteria for paraneoplastic neurological syndromes. J Neurol Neurosurg 
Psychiatry 2004;75:1135-1140.

21. Budhram A, Nicolle MW, Yang L. The positive predictive value of on- coneural antibody testing: a retrospective review. Can J Neurol Sci 2018;45:577-579. 